Special issue of the 3rd International Conference on Computational and Experimental Science and Engineering (ICCESEN 2016)

\title{
Application of Predictive Maintenance System in Drinking Water Pumping Stations
}

\author{
R. KILIÇ ${ }^{a, *}$, R. KOZAN ${ }^{b}$, D. KARAYEL ${ }^{c}$ AND S.S. ÖZKAN ${ }^{c}$ \\ ${ }^{a}$ Sakarya University, Sakarya M.Y.O. 54187 Sakarya, Turkey \\ ${ }^{b}$ Sakarya University, Engineering Faculty, Adapazari Sakarya, Turkey \\ ${ }^{c}$ Sakarya University, Technology Faculty, Adapazari Sakarya, Turkey
}

\begin{abstract}
Pumping stations are some of the most important facilities of water administration. Continuous operation of these facilities is of utter importance, to be able to serve at all times. Maintenance of these facilities is also crucial for uninterrupted operation. In this study, the functioning of the predictive maintenance technique, which is based on the vibration analysis, is briefly addressed, and the application of the technique in a real system is presented. In the real system, the steps that must be done in order to apply this system are shown step-by-step. At the end of the study, the failures determined by using the predictive maintenance method are analyzed, and solution suggestions are presented.
\end{abstract}

DOI: 10.12693/APhysPolA.132.1016

PACS/topics: 43.40.-r, 62.25.Jk

\section{Introduction}

The predictive maintenance (PM), that emerged as a result of the application of vibration management in the industry, has also attracted interest in our country, in recent years, and many enterprises have started to investigate the subject and implement this method. The aim of the study is to show that this maintenance method, which started to be used in our industry, can also be used in water management, and to show the benefits of this maintenance system for water management.

Some of the main facilities of water administrations, the pumping stations (PS), are built for the purpose of transferring water from a particular altitude to a higher altitude. Continuous operation of these stations is of great importance in terms of the continuation of the service. Thus, the maintenance management to be applied in these stations is utterly important. The purpose of the management system is to minimize the failures, and if possible, to prevent them completely and, thus, to keep the operation capacity of the system by reducing the halting period of the motopumps in PSs.

Many studies were carried out with regard to the failure detection based on the vibration analysis, which is one of the PM techniques. In their study, Akturk and Gohar [1] examined the effect of the change in ball size on the axial and radial vibrations of an axis in an axis bearing system, bedded with a bearing with two axial marbles. In his study, Bently [2] carried out research on how the thermal friction of rotor-stator would show itself at a nominal turning speed. In another study, Bently [3] stated that the estimated maintenance philosophy was

*corresponding author; e-mail: recepkilic@sakarya.edu.tr accepted in all industries, by using the knowledge of vibration, for the purpose of reducing operation cost and of increasing machine effectiveness. Bognatz [4] first defined what is an incorrect alignment and what types of incorrect alignment there are, and examined the effect of incorrect alignment on machines. Forland [5] conducted a study, showing the effect of phase angle, in order to detect machine problems. Cheng and Yang [6] conducted a study on the EMD method and Hilbert spectrum application for the failure detection of roller bearings with the pulley. Furthermore, Derakhshi and Shukur [7] conducted a study on failure detection in centrifugal pumps by using the vibration analysis method.

The most prominent property that differentiates the present study from the studies carried out so far, is the strategy of applying the PM system in PSs, and spplication of this maintenance system in the field. Furthermore, the failures that can be detected in motopumps (electrical motor and pump), using the PM system, were determined. Three failures, found by using the vibration analysis, were given as examples.

\section{Maintenance systems applied in pumping stations}

The most important objective of a maintenance system is to minimize the failure of machines, and if possible, to prevent it completely. Very frequent important failures will lead to large environmental damages in machines and will extremely increase the repairment cost [8]. The second objective of the maintenance system is to be able to guess maintenance needs in advance and to plan these needs exactly. The third objective of the maintenance system is to keep the operation capacity of the system at a maximum level, by means of reducing the halting (breaking) period of critical machines [9].

The maintenance system that is based on operating the machines until a failure occurs and having them repaired 
is called after-failure maintenance. While there is the opportunity to completely give up on this maintenance system in pumping stations in water administrations and other industrial institutions, the objective is to minimize the rate of maintenance, performed by using this maintenance system. Periodic protective maintenance is to perform the maintenance by halting (stopping) the machines at periods suggested by the producers of the machines, and if necessary, by adjusting the operation conditions [10].
Predictive maintenance is based on the principle of monitoring the performance of the machines while they are operating. Performance monitoring aims to estimate both, when machines can break down, as well as why. This maintenance technology is based on the fact that machine parts give certain types of warning before they break down. The vibration was used as the failure detection parameter, as it gives a warning of almost all typical failures, seen in pumps and electrical motors, beforehand [11].

TABLE I

Predictive maintenance parameters [12].

\begin{tabular}{|c|c|c|c|c|c|c|}
\hline \multirow[b]{2}{*}{$\begin{array}{l}\text { Cause } \\
\text { of } \\
\text { failures }\end{array}$} & \multicolumn{6}{|c|}{ Parameters } \\
\hline & Temperature & Pressure & $\begin{array}{c}\text { Leak } \\
\text { tightness }\end{array}$ & $\begin{array}{c}\text { Oil } \\
\text { analysis }\end{array}$ & $\begin{array}{c}\text { Monitoring } \\
\text { of the electric } \\
\text { current }\end{array}$ & Vibration \\
\hline Unbalance & & & & & $\sqrt{ }$ & $\sqrt{ }$ \\
\hline Misalignment & $\sqrt{ }$ & & & & $\sqrt{ }$ & $\sqrt{ }$ \\
\hline Journal bed failures & $\sqrt{ }$ & $\sqrt{ }$ & $\sqrt{ }$ & $\sqrt{ }$ & & $\sqrt{ }$ \\
\hline Gear failures & & & & $\sqrt{ }$ & & $\sqrt{ }$ \\
\hline Mechanical looseness & & & & & & $\sqrt{ }$ \\
\hline Electrical motor failures & $\sqrt{ }$ & & & & $\sqrt{ }$ & $\sqrt{ }$ \\
\hline Hydraulic and aerodynamic failures & & $\sqrt{ }$ & & & & $\sqrt{ }$ \\
\hline
\end{tabular}

\section{Faults that can be determined with the vibration analysis in PSs}

Vibration problems play an important role for correct operation and performance in electrical motors and centrifugal pumps. The reasons of the vibrations in electrical motors and centrifugal pumps may be of mechanical, fluid and electromechanical origin [12]. Continuous monitoring of the vibrations that occur in PSs is of extreme importance in terms of the performance of the PSs.

\subsection{Common faults that will occur in centrifugal pumps and electrical motors}

\subsubsection{Bearing failures}

Rolling bearins are among indispensable elements of rotating machines. Most of the failures that occur in machines (for example, electrical motors and centrifugal pumps) result from rolling bearings. For this reason, application of the PM method to electrical motors and pumps will be a great gain [8]. The periodical examination of failure occurrence and development in bearings and the replacement of the rolling bearings, when the state of the failure development reaches some limit, not only prolong the life of the rolling bearings, but also ensure a constant effectiveness and prevent a major part of the expenses, due to the reduction of early part change [8].

\subsubsection{Detection of imbalance, misalignment and mechanical looseness}

The main reasons for the imbalance in motopumps are porosity in the pump fan, inappropriate density of the material, production errors, unwanted erosion and corrosion in pump fans, coupling maladjustment, inappropriate mass distribution in the bearing arms and wings of the electrical motor, missing balance weights, and a bent shaft bearing [13-14]. Misalignment is a situation that occurs when the central axes of two or more rotating machine shafts are not on the same axis, when compared to each other [11]. Mechanical looseness occurs as either the mechanical looseness of rotating elements or the mechanical looseness of stable elements. The excessive gap between the rotating and stable elements in electrical motors and pumps is defined as rotating mechanical looseness. The gaps between stable parts, such as the basis and the motopump feet, occur as stable mechanical looseness [11].

\subsection{Faults detection in centrifugal pumps}

The vibrations encountered in pump systems can be generally classified as mechanical, hydraulic and electromechanical. And the hydraulic vibration sources, which are only inherent to centrifugal pumps, are defined as cavitation, back circulation, hydraulic imbalance and the interaction between the body and the diffuser. Stable 
and effective operation requires operation of the pump below its characteristic curve. Otherwise, the unstable flow will lead to excessive vibrations, caused by hydraulic and aerodynamic events [12].

\subsection{Vibration sources in AC motors}

We can classify the reasons of the vibration in AC motors in two groups, electrical and mechanical. Failures of mechanical origin are mentioned above. In this section, the electrical problems are the stator problems, rotor problems, eccentric rotor and thermal bending. Stator problems that can be determined by the vibration analysis in motors are stator external centeredness, short-circuit laminations, and looseness or weaknesses that can occur in the stator. The rotor problems, that can be identified by the vibration analysis, are broken or cracked rotor sticks, thermal bending of the rotor and eccentric rotor.

\section{Application of the predictive system in PSs}

When starting to apply the PM model in water administrations, it is essential to classify the motopumps in PSs, first, as very critical, critical and less critical. It is also important to gain some experience by starting the vibration monitoring system at a controllable extent, at first, and then to gradually increase the number of monitored PSs and motopumps. For a successful PM application, the motopump measurement periods should be determined in advance and should not exceed the determined period [15]. This period can be reduced accordingly for the PSs and motopumps in critical situations.

In this study, the pumping stations of Istanbul Water and Sewage Administration (ISKI) were examined oneby-one, and the PSs of ISKI were classified into three groups by their directorates in order to apply the PM model within ISKI in the best manner. Then, the PSs that are affiliated to these Directorates were classified as critical and non-critical plants. While making a classification at this stage, the function of PSs and the sizes of pumps and electrical motors were examined. While examining the pumping stations according to their functions, the population that can be affected, in case a pump in that station breaks down, was taken into consideration. At first, motopumps over $300 \mathrm{~kW}$ were included in the scope of PM, and then the motopumps in this PM were given numbers and introduced into the computer program.

Furthermore, one of the most important stages in the transition to the PM method is the determination of the vibration measurement points and measurement methods of motopumps. Determination of the measurement points before making vibration measurement on motopumps will help us both in terms of determining the best data collection points for the analysis and the determination of potential vibration sources [12]. For this reason, the nearest spots to the bedding areas were chosen as measurement points in motopumps. When choosing these points, the areas where metal is connected to the metal, painted surfaces, unloaded bearing areas, split or cracked beds and structural gaps should be avoided [16].

It is also important in the PM application to take the vibration measurements from the same spot and directions at all times, for comparison purposes and for monitoring the bending curves of motopumps [9]. Different measurement spots and directions on motopumps are shown in Fig. 1.

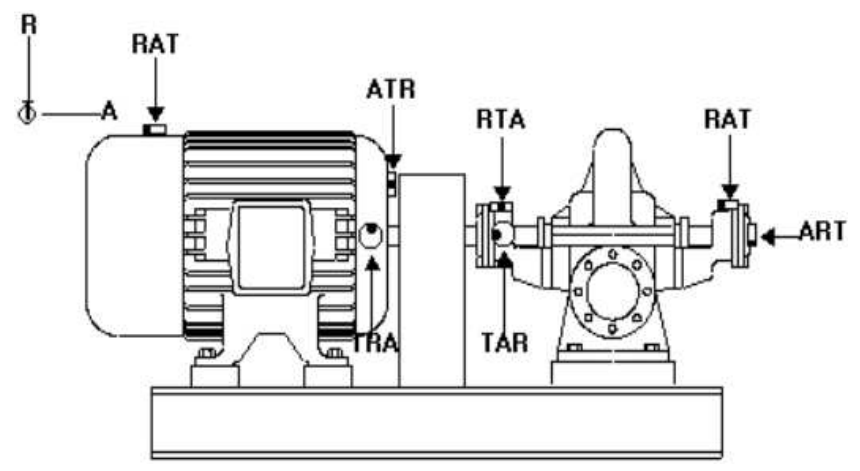

Fig. 1. Different measurement directions that can be selected on the motopump [17].

\section{Practice work}

Many failures were encountered during the application of the PM system. Three of them are presented below as examples. The failures encountered during this application were eliminated by stopping the system, and the vibrations before and after the failure were compared. What renders our study different from other studies is that it shows what kind of path to follow in the establishments, where the predictive maintenance system will be applied first and exemplifies the failures to be determined using the vibration analysis. Furthermore, this study has formed the substructure of the online monitoring of the vibrations of motopumps, which is the next step of the application.

\subsection{Case study I (determination of bearing failure)}

The example of the bearing failure given here is the vibration graph of one of the shaft bearings between the pump and motor in waste water PSs in Tuzla wastewater treatment plants. The movement transfer between the electric motor and the pump was performed by means of a shaft with the height of $30 \mathrm{~m}$, which was bedded at four places. Periodic measurements were conducted at 8 points and in 3 directions on the system. The power of the measured pump was $372 \mathrm{~kW}, 590 \mathrm{RPM}$, and electrical motor had $420 \mathrm{~kW}, 594 \mathrm{RPM}$. The main failure frequencies of the bearings are shown in the table below.

Here NB is number of bearing balls, BPFI is ball pass frequency of inner ring $[\mathrm{Hz}], \mathrm{BPFO}$ is ball pass frequency of outer race $[\mathrm{Hz}], \mathrm{BSF}$ is ball spin frequency [Hz], FTF is fundamental train frequency $[\mathrm{Hz}]$. 
TABLE II

Main failure frequencies of the bearings.

\begin{tabular}{c|c|c|c|c|c|c}
\hline \hline No. & Bearing No. & NB & BSF & FTF & BPFI & BPFO \\
\hline MS & SKF7326 & 12 & 2.52 & 0.40 & 7.15 & 4.85 \\
MK & SKF23026 & 30 & 5.54 & 0.46 & 16.34 & 13.66 \\
S1 & SKF2316 & 12 & 2.29 & 0.40 & 7.82 & 5.18 \\
S2 & SKF1316 & 15 & 2.95 & 0.42 & 8.73 & 6.27 \\
S3 & SKF2316 & 12 & 2.29 & 0.40 & 7.82 & 5.18 \\
S4 & SKF1316 & 15 & 2.95 & 0.42 & 8.73 & 6.27 \\
PK & SKF7326 & 12 & 2.52 & 0.40 & 7.15 & 4.85 \\
PS & SKF23026 & 30 & 5.54 & 0.46 & 16.34 & 13.66
\end{tabular}

Upon examining the graphs, it is seen that a peak of $3.89 \mathrm{~mm} / \mathrm{s}^{2}$ is formed at $41.44 \times \mathrm{RPM}$ of the high band spectrum, in the axial direction of the shaft bearing number three. This shows us that it is one of the harmonics of BPIF, which is one of the main failure frequencies. The formation of the side bands of BPIF in the axial direction shows that the failure has advanced (Fig. 2). Upon examining the time graphs of the vibrations taken from the bed, the formation of periodic strikes is a sign of typical bearing failure (Fig. 3). The advancement process of the failure is seen in Fig. 4.

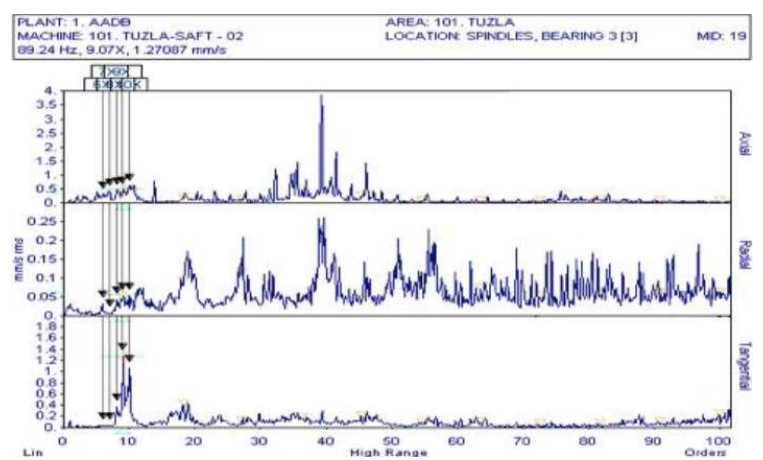

Fig. 2. Three-directional spectrum of the shaft bearing bed.

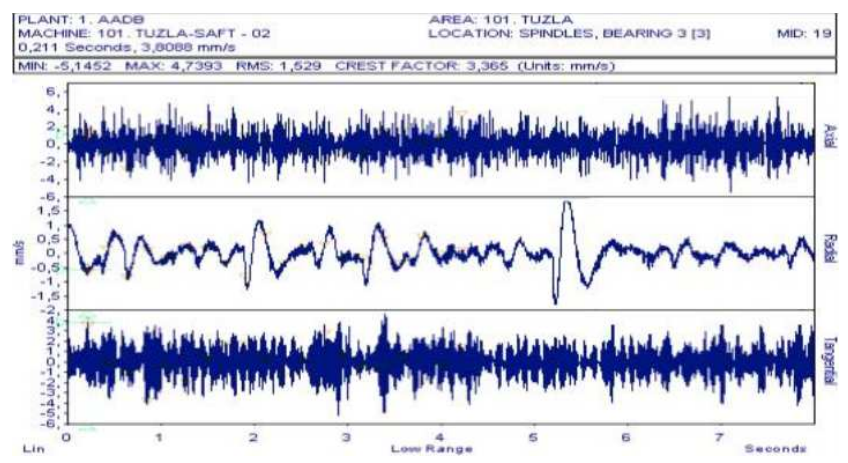

Fig. 3. Waveform (time) graph in three-dimensions of the shaft bearing bed.

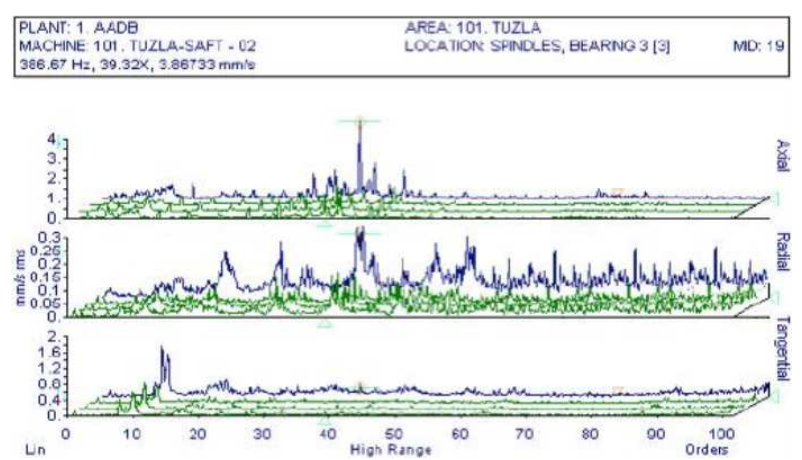

Fig. 4. Comparison of spectra of the shaft bearing bed.

\subsection{Case study II (detection of failure of hydraulic origin)}

The least encountered type of problems in PS, which we have addressed within the framework of the PM, is problems of hydraulic origin. Problems of hydraulic origin were only encountered in Buyukcekmece Clean Water Pumping Station, among the facilities that we included in the PM system and vibration measurements of which were taken periodically. The parameters of the electric motor in this facility are $2000 \mathrm{~kW}, 1484 \mathrm{rpm}$, and parameters of the pump are $1645 \mathrm{~kW}, 1484 \mathrm{rpm}$.

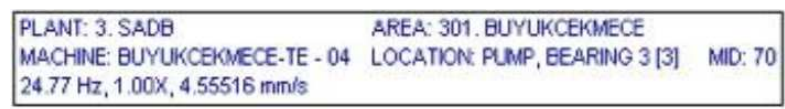
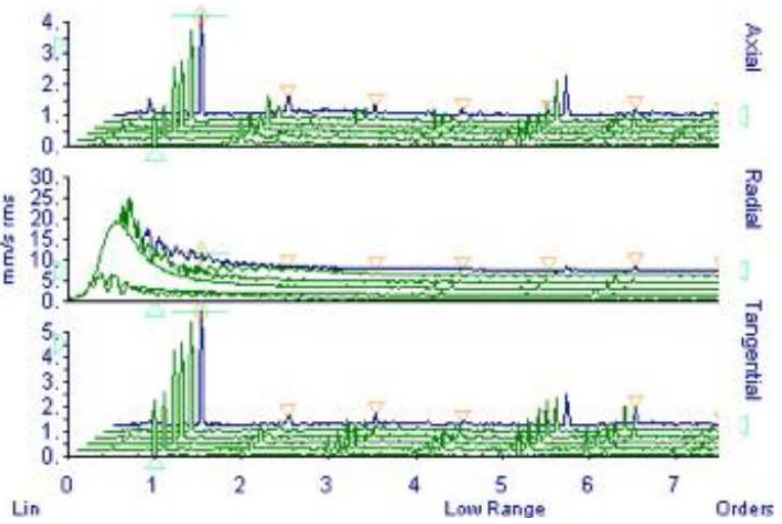

Fig. 5. Comparison of spectra of the coupling side of the pump.

There is a peak of high amplitude under the motor rotation, in the vibration graph, taken from the pump coupling, in the radial direction (Fig. 5). This peak has the form of a resonance, and has given us, at first, the suspicion that the system entered into resonance. As a result of these periodic measurements we saw, that this peak in the radial direction is always present and that it has an amplitude of $3.19 \mathrm{~mm} / \mathrm{s}^{2}$ in the axial direction and an amplitude of $4.5 \mathrm{~mm} / \mathrm{s}^{2}$ in the tangential direction. 
Upon looking at the vibration graphs in other dimensions, we saw that the incident does not only result from resonance but the water intake structure of the pump or the irregularities in the flow.

For the definite determination of this problem, the vibration graphs of the coupling side and free side of the pump should be examined, considering all of the measurements in three directions. There are vibration peaks at both the motor rotation and pump fan transmission frequency in vibration graphs of the coupling side, as well as of the free side (Fig. 5 and 6), which show that the incident results from turbulence, back circulation or the gap between the diffuser and the body. When the water intake structure of the pump was examined, it was considered that the back circulation incident may have happened.

\begin{tabular}{|lll|}
\hline PLANT: 3. SADB & AREA: 301. BUYUKCEKMECE & MO . \\
MACHINE: BUYUKCEKMECE-TE - 04 & LOCATION: PUMP, BEARNG 4 [4] & MO: 70 \\
$24.70 \mathrm{~Hz}, 1.00 \mathrm{X}, 0.91525 \mathrm{~mm} / \mathrm{s}$ & & \\
\hline
\end{tabular}

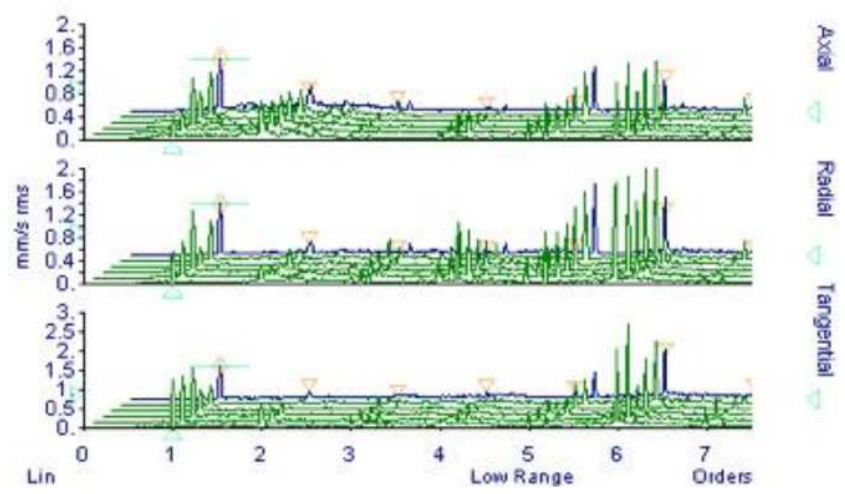

Fig. 6. Comparison of spectra of the free side of the pump.

Since the pump gave vibration graphs of the same character when it operated alone, this incident did not exist. The fact that $1 \times \mathrm{RPM}$ harmonics exists in the vibration graphs in all three directions shows, that the incident may result from mechanical looseness. As a result of the performed studies, it was found that this amplitude of $20-25 \mathrm{~mm} / \mathrm{s}^{2}$ in the radial direction resulted from the turbulence that occurred in the fluid, as the gap between the diffuser and body was not eccentric. Vibration measurements were made after the gap adjustment between the diffuser and body had been performed. In the measurements taken, it is seen that vibration amplitudes are reduced to $9.19 \mathrm{~mm} / \mathrm{s}^{2}$ in the radial direction.

\subsection{Case study III (determination of the rotor failure in electric motor)}

Vibration measurements were taken from a motor of 4 poles, performing 1500 rotations $/ \mathrm{min}$. The measurements were made in the tangential, radial and axial directions from the motor free side and from the motor coupling side. As it can also be seen in the graphs, the vibration amplitudes gradually increase in the radial and tangential directions and the vibration amplitudes occurring at $1 \times$ line frequency and $2 \times$ line frequency are the most obvious signs of thermal bending. In addition to this, the vibration peaks with sliding frequency are another sign of such failure. The stator magnetic area frequency is $1500 \mathrm{rpm}$, and the frequensy of the rotation of rotor is $1476 \mathrm{rpm}$. In the obtained vibration graphs, frequency of $24 \mathrm{~Hz}$, which is the sliding frequency in both, the radial and tangential directions, and the vibration peaks in its harmonics can be clearly seen. The figures below (Figs. 7 and 8) demonstrate the change of the graphs taken from both bed sides of the motor, and the motor vibrations, in time.

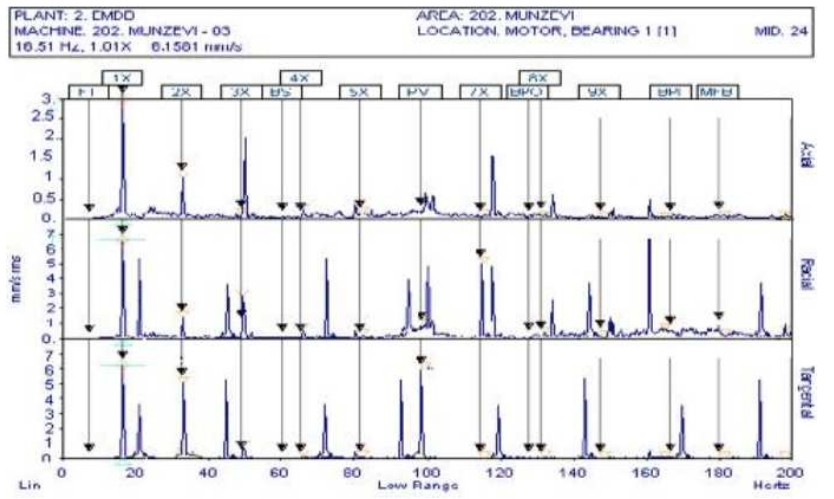

Fig. 7. Spectrum from the motor free side.

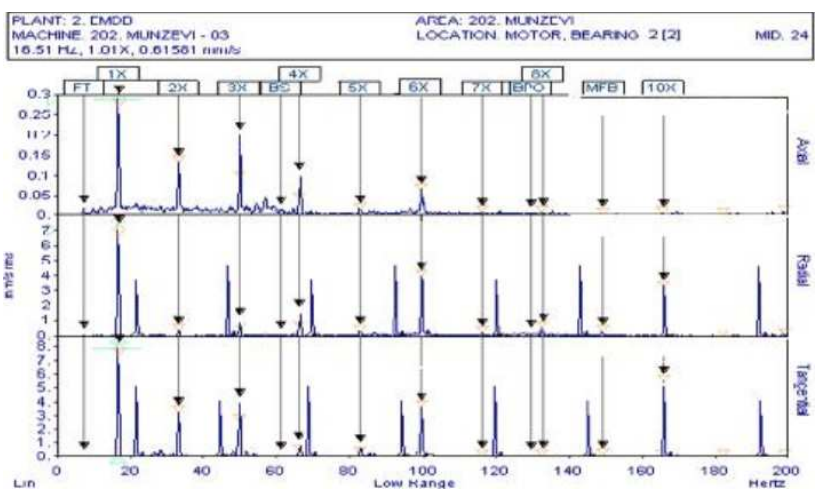

Fig. 8. Spectrum from the motor coupling side.

\section{Conclusions}

In this study, the criteria needed for the transition to the PM technique in the enterprises, where the PM technique is not used, were primarily examined. The things that should be done for the application of the PM technique in an enterprise were addressed separately. It was specified in which machines the PM technique should be applied in enterprises, how to choose the measurement points in these machines, and what measurement directions in the selected measurement points should be used. As a result of this study; 
- The measurement points and measurement directions in the motopumps should be the same in all mesurements. The input and output pressures in motopumps should be the same as the input and output pressure during the previous measurement. If the motopumps at the right and left side of the given motopump were in the operating state in the previous measurement, they should be in the operating state during the following measurements.

- Stable speed sensors should be placed at the points where vibration measurement will be made, during the production of the motopump.

- While deciding on any failure in the motopumps, it is necessary to look at the vibration amplitude in failure frequencies and its harmonics instead of the general vibration values.

- Vibration measurements should be performed at specific periods in order to achieve an effective failure detection in motopumps. Any intervention into the motopumps should be definitely recorded, and these changes should be taken into consideration during the vibration analysis.

- Other PM parameters, alongside the vibration measurements, should be used in enterprises.

- The PM parameters in enterprises should be monitored online by establishing central supervision systems.

- As a result of this study, it was decided to implement such system in ISKI, the institution where this study was carried out.

\section{References}

[1] N. Aktürk, R. Gohar, Proc. Instn. Mech. Engrs. Part J 212, 101 (1998).

[2] D.E. Bently, Basic Rotor-to-Stator Thermal Rubs which Exhibit Rotative Speed (1×) Symptom Only, www.bentlicom/articls/996don.asp, Orbit, September 1996.
[3] D.E. Bently, Predictive Maintenance Through the Monitoring and Diagnostics of Rolling Element Bearing, Applications Note www.bentlicom/articls/apnotles/ an044pxa.asp, 2001.

[4] S.R. Bognatz, Alignment of Critical and Noncritical Machines, www.bently.com/service/training/ mlmidalign.htm, 2000.

[5] C. Forland, Why Phase Information is Important for Diagnosing Machinery Problems, www.bentli.com, Orbit, September 1999.

[6] D. Yu, J. Cheng, Y. Yang, Mech. Syst. Sign. Proc. 19, 259 (2005).

[7] M.R.F. Derakhshi, Z. Shukur, Informatica 35, 259 (2011).

[8] N. Aktürk, M. Uneeb, R. Gohar, J. Tribol. 119, 747 (1997).

[9] P. Liggan, D. Lyons, Pharmaceutical Engin. 31, 1 (2011).

[10] A. Almasi, Australian J. Mechan. Engin. 14, 199 (2016).

[11] D.W. Gelen, Introduction to Machine Vibration, DLI Engineering Corp., 1993.

[12] A.R. Crawford, S. Crawford, The Simplified Handbook of Vibration Analysis, Computational Systems Inc., 1992.

[13] J.I. Taylor, The Vibration Analysis Handbook, Vibration Consultants, 1994.

[14] W. Victor, Machinery Vibration Measurement and Analysis, McGraw Hill Book Company, 1991.

[15] S. Orhan, N. Aktürk, V. Çelik, NDT E International 39, 293 (2006).

[16] S.M. Abdel-Rahman, A.A. El-Shaikh Sami, Diagnosis Vibration Problems of Pumping Stations: Case Studies in: 13th Int. Water Technology Conf., IWTC 13, Hurghada, Egypt 2009.

[17] R. Kilic, Acta Phys. Pol. A 130, 487 (2016). 